\title{
Application of Fuzzy Multi-Objective Decision Making in Spatial Load Forecasting
}

\author{
Mo-yuen Chow \\ Department of Electrical and Computer Engineering \\ North Carolina State University \\ Raleigh, NC 27695-7911
}

\begin{abstract}
Electric distribution system planning is to provide an economic expansion plan to meet the future demands in its territory. A forecast of the future electric demand and its geographic distribution is a prerequisite for distribution planning. The quality and accuracy of this forecast have large influence on the quality of the electrical distribution system planning. Spatial load forecasting emerges to provide a more accurate prediction of both the magnitudes and locations of future electric loads. Since the load growth pattern is dominated by its land-use (residential, commercial, or industrial), the land usage study of small area is important to capture the future loads accurately. There are many factors which will affect the customer land-use decision, for example, distance to highway, distance to urban pole, and the costs. The customer's preferences can be estimated based on these objective factors. Then the land utilization and the electricity consumption can be estimated. Since the objectives sometimes are conflicting each other, it can be cumbersome to use conventional cost function approach to determine the land usage decision. This paper will apply fuzzy multiobjective decision making scheme to the urban redevelopment and spatial load forecasting, which is more naturally and straight forward used to handle the spatial load forecasting problem. An example is used to illustrate the proposed methodology.
\end{abstract}

Keywords: Spatial load forecasting, land-usage, urban redevelopment, fuzzy logic, Yager multi-objective decision making, information technology

\section{Introduction}

Distribution systems aim to provide reliable power to customers in a large geographic areas. In the planning stages, utilities need to plan ahead for anticipated future load growth under different possible scenarios. Based on load forecasts, they will decide whether to built new facilities or upgrade the existing facilities. Their decision can affect the earning or losing millions of dollars for their companies as well as customers' satisfaction and operational reliability. Therefore, decision making tools are important to make a right decision based on given information. The correct plan will rely on the accurate load forecasting.

For distribution planning, not only the load magnitude but also its location are to be predicted. The load in distribution level is highly stochastic ('needle peaks') and greatly affected by land usage, weather, and living habits. Apparently, the

PE-965-PWRS-2-06-1997 A paper recommended and approved by the IEEE Power System Planning and Implementation Committee of the IEEE Power Engineering Society for publication in the IEEE Transactions on Power Systems. Manuscript submitted November 18 , 1996; made available for printing June 9, 1997.

\author{
Hahn Tram \\ Distribution Information Systems \\ ABB Power T\&D Company Inc. \\ Cary, NC 27511
}

distribution load forecasting is a high dimensional, stochastic, nonlinear, and time varying problem. There are few successful either mathematical models or statistical regression models for distribution load forecasting.

There are a lot of unforeseen situations may occur and land usage may change through time. For example, the new construction of a highway, the move in of a large industry plant. These external factors can substantially affect the land usage, thus the load growth. The distribution system planners need to aggregate different types of information to predict what might happen in their service areas in the future and plan accordingly. Load studies shows that the land usage dominate the load growth pattern and load shapes because they employ similar type of appliances and have similar needs and schedule [1].

The land usage based spatial load forecasting computer simulation has been proposed and used to aggregate appropriate geographic information to simulate future load growth based on different anticipated scenarios [2]. The increasingly popular, affordable, and accurate Geographic Information Systems (GIS) technology provide an excellent data base platform for spatial load forecasting techniques. The use of GIS can save thousands of man hours for utilities to collect relevant geographic data [3]. Thus spatial load forecasting technology become even more attractive than before both from economical point of view and superior load forecasting accuracy.

There are a few stages for spatial load forecasting as shown in Figure 1. The spatial information is used to predict the land usage. Each land usage is mapped to a load growth pattern. The land usage and load growth are then calibrated based on different constraints, such as system load growth, budget available, future economy growth of the area, etc.

\begin{tabular}{|c|c|c|} 
geographic \\
information
\end{tabular} constraints $\begin{gathered}\text { land usage } \\
\text { decision }\end{gathered} \rightarrow \begin{gathered}\text { load growth } \\
\text { estimation }\end{gathered}$

Figure 1. diagram of land-use based spatial load forecasting.

Spatial load forecasting techniques have been shown to provide superior results than other distribution load forecasting methods such as regression [4]. Land-use based spatial load forecasting simulation program is basically a tool to select land usage and project the future loads under different scenarios. This is a typical decision making problem. Generally, heuristic intuition, expert knowledge and experience, and linguistic descriptions are very important for engineers to evaluate the results. Since some information only can be described imprecisely and some others only as quality, the decision making environment is fuzzy. The conventional 
multi-objective decision making scheme does not capture imprecise information and quality data in an effective manner.

As shown in Figure 1, land usage decision making is an important part for spatial distribution load forecasting. [7, 8] has reported the feature map approach for spatial load forecasting. [9] has introduced the Fuzzy Logic techniques to solve the spatial load forecasting land usage selection. This paper will focus on using Fuzzy Multi-Objective Decision Making techniques to solve Spatial Load Forecasting problems.

\section{Spatial Load Forecasting Modeling}

\subsection{Spatial Load Forecasting Problem}

As discussed in [10], three kinds of information are involved in the decision process : Goals, Constraints, and Alternatives. For the spatial load forecasting problems, we have to identify them appropriately.

Goals are what we want to achieve out of the decision process. In the land usage spatial load forecasting program, distribution engineers want to predict the likelihood of land usage changes in the future due to different influential factors, then estimate the spatial load growth patterns accordingly under different scenarios in order to plan the distribution system ahead of time. The land usage goals can be further categorized as :

1. Determine whether the land needs re-development [7],

2. Determine what land class the site will become if redevelopment is required, and

3. Determine the corresponding load growth for the land usage.

Constraints/criteria are limiting factors that are needed to consider before deciding how to achieve the goals. The land usage constraints/criteria can be Land-use Preferences, Budget Limitations, Geographic Constraints, etc.

Alternatives in the land usage decision are the available choices for land usage under consideration. For example, the alternatives are different land class usage: Vacant land, Light Residential, ..., Heavy Industry.

\subsection{Issues Under Considerations}

In order to use fuzzy logic technologies to solve the problem, there are three major issues needed to be addressed:

1. How to implement linguistic descriptions of the land-use selection and load forecasting?

2. How to aggregate the available information for decision making?

3. How to infer the final decision based on the aggregated information?

In this paper, three popular fuzzy logic techniques are used to answer the issues posted. Membership functions will be used to convert the input values to the linguistic descriptions and membership values. The $\min$ fuzzy inference is used to aggregate inputs and fuzzy rules. Centroid rule, which is the popular method to perform defuzzification in fuzzy logic
[11], will be used to evaluate the fuzzy outputs into an crisp preference value for each alternatives for decision making. Yager's multi-objective decision technique $[10,12]$ will then be used to infer the final decision making based on the preference value obtained from the Centroid rules and the weightings of objectives. The overall decision process is shown in Figure 2.

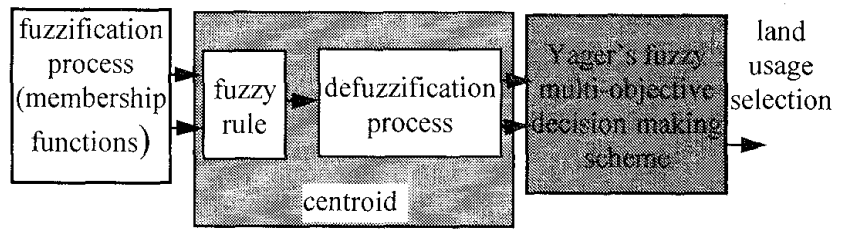

Figure 2. Fuzzy multi-objective decision making process

Fuzzy logic technology is a rich field with a large amount of theory and operations developed [11]. This section briefly describe the techniques in fuzzy logic, including membership functions, fuzzy rules, fuzzification, defuzzification, and fuzzy multi-objective decision making in order to facilitate our future discussion. For more detailed information about fuzzy logic technology, please refer to [13].

\subsection{Fuzzy Sets/Membership Functions and Fuzzification}

A fuzzy set is a set containing elements that have varying degree of membership in the set. Elements of a fuzzy sets are mapped to a universe of membership values using a functiontheoretic form and is termed membership function [10]. There are different ways to come up with membership functions. Subjective judgment, intuition and expert knowledge are commonly used in constructing membership functions.

Linguistic terms such as Close and Far are often used to describe distance. Distance from highway is an important factor for many buyers to determine whether the site is suitable to be used as a residential site, commercial site, or industrial site. Since the descriptions of many linguistic terms are relative in nature, we need to define the range of the membership functions of the linguistic variable to cover. The range is termed the universe of discourse of the membership function. The membership values of each functions are usually normalized between 0 and 1 , where 0 indicates nonmember and 1 indicates full member of the membership function respectively. Figure 3 shows the membership functions of three linguistic variables: Very Close $(V)$, Moderately Close (C), and $\operatorname{Far}(F)$ used to describe the distance from the highway. For example, 1 miles has 0.5 membership value of being moderately close while 0.5 membership value of being very close.

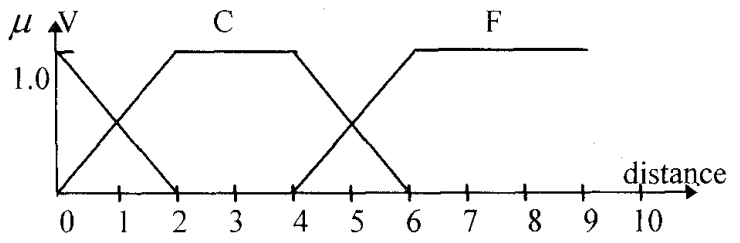

Figure 3. Distance membership functions.

Even though the choices of membership functions are subjective, there are still some rules of thumb for membership 
function selection that can produce good results [5]. In general, we would like to choose the membership functions which overlap other neighboring membership functions by 25-30\%. In addition, we would like to select sensible membership values.

\subsection{Fuzzy Rules}

Heuristic and expert knowledge are often expressed linguistically in the form of If-Then rules. These rules can be extracted from common senses, intuitive knowledge, survey results, general principles and laws, and other means that reflect actual situations. For example, the average home buyer generally prefers to buy a house that is close to highways for the convenience of commuting, yet not too close to highways in order to avoid the noises and air pollution generated from the highways. The rules for selecting residential site with respect to distance to highway can then be described as:

1. If the distance is very close to the highway, then strongly against.

2. If the distance is moderate close to the highway, then strongly prefer.

3. If the distance is far from the highway, then moderate against.

\subsection{Spatial Load Forecasting Description}

One of the major process in the land-use based spatial load forecasting relies on the prediction of future land usage. The choice of land usage belongs to the multi-objective decision evaluation problem that is based on different factors. The typical multi-objective decision problem, which basically a decision process, involves the selection of one alternative, $a_{i}$, from a universe of $n$ alternatives $A=\left\{a_{1}, a_{2}, \cdots, a_{n}\right\}$ given a set of $r$ objectives/criteria $O=\left\{o_{1}, o_{2}, \cdots, o_{r}\right\}$ that are important to the decision making. Each alternative will be evaluated on how well it satisfies each objective.

Distance to highway concept is straight forward [1]. Urban pole concept has been used in city planning and modeling [14]. Among a city or town, site preference may attracted to or repulse from some salient point of geographic interests such as center of district, shopping centers, ball parks. The influence of the center of interest is often presented by the Urban Pole concept [1].

For example, there are three alternatives - residential, commercial, and industrial - are considered for land-use selection in the spatial load forecasting problems [9], that is, $n=3$ and $A=\left\{a_{1}, a_{2}, a_{3}\right\}$. Suppose two objectives are considered - $O_{1}$ distance from highway and $O_{2}$ distance from urban pole - then $r=2$ and $O=\left\{O_{1}, O_{2}\right\}$.

\subsection{Yager's Fuzzy Logic Multi-Objective Decision Making Scheme}

Each alternative will have a crisp output value after the defuzzification method [12]. In the multi-objective decision making process, each objective has its relative weighting with respective to others. In 1981, Yager proposed an approach for decision making that required only ordinal information on the ranking of preferences and importance weights [10]. This process naturally requires subjective information from the decision authority concerning the importance of each objective. Based on the multi-objective decision making formulation described previously, the decision measure for a particular alternative, $a$, can be replaced with a classical implication of the form [12],

$$
M\left(O_{i}(a), b_{i}\right)=b_{i} \rightarrow O_{i}(a)=\bar{b}_{i} \vee O_{i}(a) .
$$

where $\overline{b_{i}}=1-b_{i}$ and $\vee$ is the $\max$ operator, i.e.,

$$
\bar{b}_{i} \vee O_{i}(a)=\max \left\{\bar{b}_{i}, O_{i}(a)\right\} \text {. }
$$

The implication preserves the linear ordering required of the preference set, and at the same time relates the two quantities in a logical way where negation is also accommodated. Justification of the implication as an appropriate measure can be developed using an intuitive argument [10]. A reasonable decision model will be the joint intersection of $r$ decision measures,

$$
J=\bigcap_{i=1}^{r}\left(\bar{b}_{i} \cup O_{i}\right)
$$

and the optimum solution, $a^{*}$, is the alternative that maximizes $J$. If we define

$$
C_{i}=\bar{b}_{i} \cup O_{i}
$$

hence $\mu_{C_{i}}(a)=\max \left[\mu_{\bar{b}_{i}}(a), \mu_{O_{i}}(a)\right]$,

then the optimum solution. expressed in membership form, is given by :

$\mu_{D}\left(a^{*}\right)=\max _{a \in A}\left[\min \left\{\mu_{C_{1}}(a), \mu_{C_{2}}(a), \cdots, \mu_{C_{r}}(a)\right\}\right]$

Yager's decision making requires users to rank the group of goals and the group of constraints along a comparative scale of importance from 0 to 1 (create fuzzy membership functions for each inputs and outputs). Then measure each of the user's alternatives against each of the goals and constraints and rank them from 0 to 1 (another membership function concept).

The preference weighting factors, $B=\left\{b_{1}, b_{2}, \cdots b_{r}\right\}$, will be assigned to each of the objectives to quantify the decision maker's feelings about the influence that each objective would have on the chosen alternative. They are used to convert the multiple objectives into an overall decision function in some plausible way. The negation of the preference weighting $\bar{b}_{j}$ acts as a barrier such that all distinctions less than that barrier is disregarded while those distinctions above the barrier is kept. The more important is the objective, the lower is the barrier, and thus the more level of distinction there are. 
In the previous example, the preference weighting factors $B=\left\{b_{1}, b_{2}\right\}$ are the user defined preference on the objectives $\left\{\mathrm{O}_{1}, \mathrm{O}_{2}\right\}$ and indicate the relative importance of each objectives toward the decision making process.

\section{Implementation of Land-use Selection}

This section presents a land usage based spatial load forecasting prototype demonstration of using fuzzy logic decision making scheme of the land usage determination, from which predicts the future spatial load growth.

\subsection{Multi-Objective Decision Problem Set-Up}

As mentioned in previous sections, the illustration problem is formulated as following:

A $10 \times 10$ land grid sites assuming all environmental conditions are the same except the distance to the highway, which is under construction, and distance to the urban pole center as shown in Figure 4. There are three alternatives for land usage - residential, commercial, industrial.

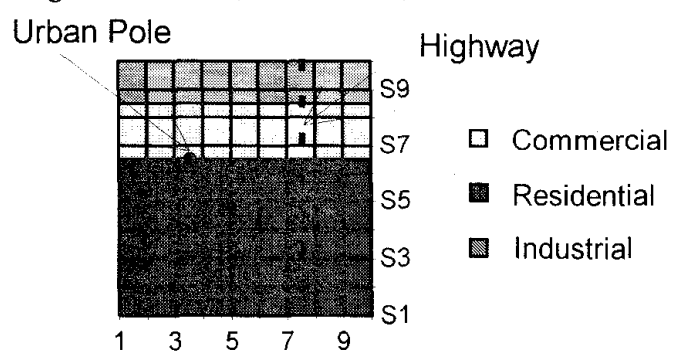

Figure 4. The illustrative example

The goals of the land usage selection are :

1. To maximize the land value by satisfy the preference of decision makers.

2. To minimize the redevelopment costs.

The inputs for the decision making process is :

1. The distance of the site to the highway.

2. The distance of the site to the urban pole.

3. The original land-use information.

4. The cost of redevelopment from one land-use to another.

\subsection{Membership Function Set-up}

The preferences of the land usage depends on the two external factors: distance to highway, $D_{h}$, and distance to urban pole, $D_{u}$. The distance to highway and distance to urban pole are described by linguistic variables : very close $(V)$, moderately close $(C)$, and far $(F)$. The membership functions representing these variables have been shown in Figure. 3. Since the grid size under consideration is $10 \times 10$, therefore, the universe of discourse of the input variable is $[0$, $10]$. The preference values are normalized between $[0,1]$, in which 1 indicates completely prefer and 0 indicates completely against.
Figure 5 shows five membership functions to describe the different site preference: strongly against (SA), moderately against (MA), neutral (NT), moderately prefer (MP), strongly prefer (SP). Again, the preference membership functions are normalized between $[0,1]$.

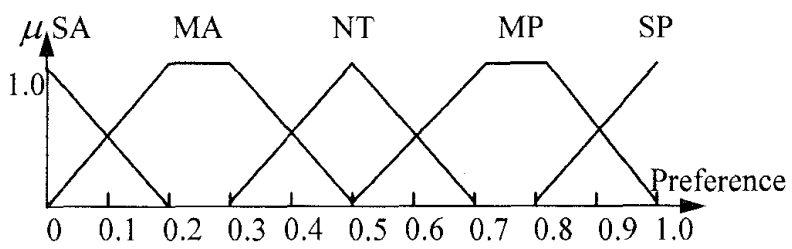

Figure 5. Preference membership functions.

\subsection{Fuzzy Rules}

The rules for selecting land usage with respect to distance to highway and urban pole can be described by the linguistic variables in the Table 1,2 . It means 'If a site is very close to highway, then residential choice will be moderately against'.

Table 1: Fuzzy rules for the distance to highway

\begin{tabular}{|c|c|c|c|}
\hline Highway & Residential & Commercial & Industrial \\
\hline Very Close & MA & SP & SP \\
Close & SP & NT & SA \\
Far & MA & MA & SA \\
\hline
\end{tabular}

Table 2: Fuzzy rules for the distance to urban pole

\begin{tabular}{|c|c|c|c|}
\hline Urban Pole & Residential & Commercial & Industrial \\
\hline Very Close & SA & SP & SA \\
Close & MP & MP & MA \\
Far & MA & MA & SP \\
\hline
\end{tabular}

Not only the distance to highway and urban pole, but also redevelopment cost is considered in land-use selection. The redevelopment cost is listed in Table 3 . Since cost is to be minimized, the preference of redevelopment is defined as $1-T_{i j}$, where $T_{i j}$ is the cost of redevelopment from $i$ th landuse to $j$ th land-use.

Table 3: The redevelopment costs

\begin{tabular}{|c|c|c|c|}
\hline & Residential & Commercial & Industrial \\
\hline Residential & 0.0 & 0.2 & 0.3 \\
Commercial & 0.3 & 0.0 & 0.4 \\
Industrial & 0.3 & 0.2 & 0.0 \\
\hline
\end{tabular}

The importance weighting factor of each objective $b$ is set differently based on the decision maker's preference. For example, highway: $b_{1}=0.7$, urban pole: $b_{2}=0.6$, cost: $b_{3}=0.5$, that means highway criterion is more important than the urban pole criterion while urban pole objective is more important than the cost criterion.

The centroid rule is applied to defuzzify the preferences to highway and urban pole. These preferences and the preference of redevelopment on cost issue are aggregated by their important weighting factors based on the Yager's approach. 
For example, site $(\mathrm{S} 6,5)$ is a residential site which is 1.5 miles away from highway and 2.55 miles from urban pole.

- fuzzification to get $\widetilde{D}\left(d_{H}\right)=\{V / 0.25, C / 0.75, F / 0\}$

- apply all applicable rules $\widetilde{P}_{R}=\{M A / 0.25, S P / 0.75\}$

- defuzzification to get a crisp preference $p_{R}=0.8125$

Where $\widetilde{D}, \widetilde{P}_{R}$ are fuzzified distance to highway and preference to residential, $p_{R}$ is the defuzzified preference. Same procedure will evaluate other two alternatives and get:

$$
\begin{array}{ll}
\widetilde{P}_{C}=\{S P / 0.25, N T / 0.75\} & p_{C}=0.625 \\
\widetilde{P}_{I}=\{S P / 0.25, S A / 0.75\} & p_{I}=0.25
\end{array}
$$

where $\widetilde{P}_{C}, \widetilde{P}_{I}$ are fuzzy preference to commercial and industrial, $p_{C}, p_{I}$ are crisp preference. Similarly to the urban pole and cost, the results are listed in Table 4. Each alternative is evaluated based on Eq. (1-6) and the highest rank win. Conclusion: this site is best served by residential.

Table 4. Results for Land-use selection

\begin{tabular}{|c|c|c|c|c|}
\hline & weight & Res. & Comm. & Ind. \\
\hline Highway & 0.7 & 0.8125 & 0.625 & 0.25 \\
\hline Urban Pole & 0.6 & 0.75 & 0.75 & 0.25 \\
\hline Cost & 0.5 & 1.0 & 0.8 & 0.7 \\
\hline Rank & & 0.75 & 0.625 & 0.3 \\
\hline
\end{tabular}

\section{Results and Discussion}

\subsection{Land-use selection}

The results of land-use redevelopment is shown in the Figure 6.
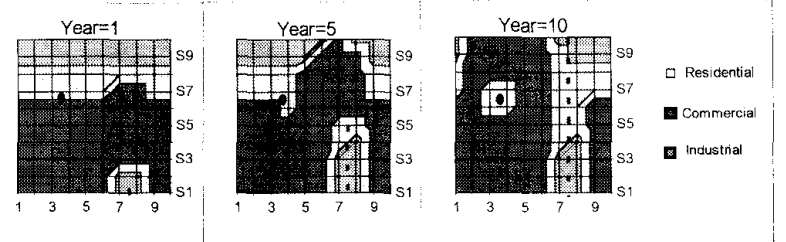

Figure 6 . The predicted land-use map in year 5 and 10

Based on the land-use selection rules, the sites around urban pole are the most preferred place for commercial. Therefore some residential sites, which are close to urban pole, are re-developed to commercial sites. On the other hand, some commercial sites and industrial sites which are neither very close nor far to urban pole nor highway are changed to residential sites. Since industrial sites are strongly against to be close to the urban pole but strongly prefer to be close to highways, the sites on the side of highway which is close to urban pole are re-developed to commercial sites while those far away from urban pole are re-developed to industrial sites. These results are consistent with the fuzzy rules and membership functions used.

\subsection{Spatial Load Forecasting}

Different loads have their own characteristics and enduser patterns. Reasonable approximations and simplifications have been studied on both load growth and end-user patterns $[1,15]$. These techniques have been used in several power areas such load modeling and characterization, and demand side load management.

In this paper, each land use has its own end-user load growth pattern and is described by state-space description in the form of :

$$
\dot{S}=a S+b,
$$

with appropriate units. The parameters for different land usage used in this paper are listed in Table 5.

Table 5. Load growth parameters used in the illustration.

\begin{tabular}{c|ccc} 
& $\mathrm{R}$ & $\mathrm{C}$ & I \\
\hline$a$ & $\left\{\begin{array}{c}0.75 \text { if } S<c \\
-0.75 \text { if } S \geq c\end{array}\right.$ & $\left\{\begin{array}{c}0.4 \text { if } S<c \\
-0.4 \text { if } S \geq c\end{array}\right.$ & $\left\{\begin{array}{c}0.6 \text { if } S<c \\
-0.6 \text { if } S \geq c\end{array}\right.$ \\
$b$ & $\left\{\begin{array}{c}0 \text { if } S<c \\
7.5 \text { if } S \geq c\end{array}\right.$ & $\left\{\begin{array}{l}0 \text { if } S<c \\
6 \text { if } S \geq c\end{array}\right.$ & $\left\{\begin{array}{c}0 \text { if } S<c \\
12 \text { if } S \geq c\end{array}\right.$ \\
$c$ & 5 & 7.5 & 10 \\
$S_{0}$ & 0.5 & 0.5 & 0.5
\end{tabular}

The load growth pattern described in Eq. (7) has been shown to be a good approximation for many load growths observed in the past. The parameters $a, b, c$ can be fine tuned to suit the specific problems at hand [1].

Once the land-use redevelopment plan has been provided, the spatial load forecasts are easy to obtain. For example, site $(\mathrm{S} 4,7)$ will be redeveloped from residential to commercial in the third year. After redeveloped it will follow the growth pattern for commercial instead of residential. In Figure 7 , different land-use has different load growth pattern. Site $(\mathrm{S} 1,3)$ is residential, site $(\mathrm{S} 6,3)$ is commercial, site $(\mathrm{S} 10,8)$ is industrial site. Due to the land-use redevelopment, the load will change accordingly. Based on the spatial load forecast results, distribution planning software can provide the best feeder design in the future.

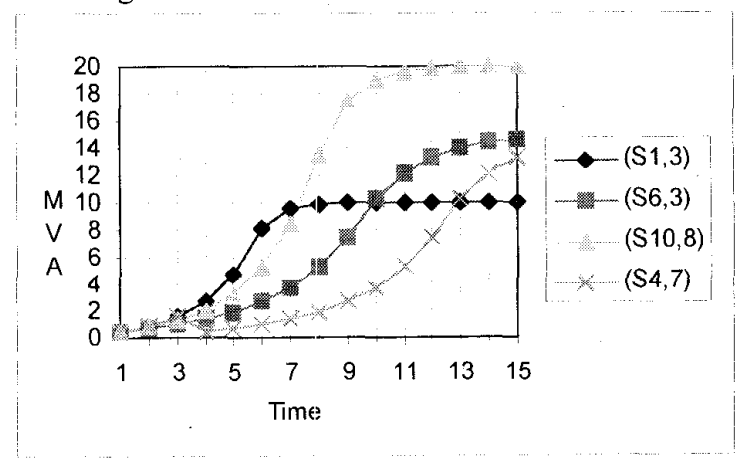

Figure 7. The electricity consumption

\subsection{The advantages of the proposed approach}

The fuzzy logic formulation provides an intuitive and convenient approach to implement heuristic rules into the 
spatial load forecasting land-use selection criteria. The fuzzy algorithm is robust even with uncertainties. In this paper, the decision is made based on the compromise of preference to highway, urban pole, and redevelopment cost. Yager's approach is used to evaluate multi-objective by the importance weighting factors. The proposed approach can also easily match the decision maker's expectation, in other word, the fuzzy rules and membership functions can be modified to fine tune the results.

\section{Conclusion}

Land-use based spatial load forecasting provides not only load magnitude but also load distribution in distribution systems. In this paper, Yager's fuzzy multi-objective decision making approach has been extended to solve the multiobjective land usage selection problems. The results shows that this fuzzy approach is robust, flexible, and easy to fine tune to get good results. The proposed method is very suitable to select land usage based on the preference of the decision maker, thus predict future load growth.

\section{Reference}

[1] H. L. Willis and G. B. Rackliffe, Introduction to Integrated Resource T\&D Planning: Automated Distribution Division, ABB 1994.

[2] H. L. Willis and T. W. Parks, "Fast Algorithms for Small Area Electric Load Forecasting," IEEE Transactions on Power Systems, vol. PAS-102, pp. 1111 7, 1983.

[3] ESRI, Understanding GIS, 6 ed: ESRI, 1992.

[4] H. L. Willis and J. E. D. Northcote-Green, "Comparison Tests of Fourteen Distribution Load Forecasting Methods," IEEE Transactions on Power Apparatus and Systems, vol. PAS-103, pp. 1190-1197, 1984.

[5] M.-y. Chow, "Fuzzy Logic Based Control," in CRC Press Industrial Electronics Handbook, D. Irwin, Ed., 1995.

[6] M.-y. Chow, "Fuzzy Systems," in CRC Press Industrial Electronics Handbook, D. Irwin, Ed.: CRC, 1996.

[7] M.-y. Chow and H. Tram, "Application of Urban ReDevelopment Methodology in Spatial Load Forecasting," submitted to IEEE Transactions on Power Systems, 1996.

[8] M.-y. Chow and H. Tram, "Methodology of Urban ReDevelopment Considerations in Spatial Load Forecasting," accepted for publication in IEEE Transactions on Power Systems, 1996.

[9] M.-y. Chow and H. Tram, "Applications of Fuzzy Logic Technology for Spatial Load Forecasting," accepled for publication in IEEE Transactions on Power Systems, 1996.

[10] T. J. Ross, Fuzzy Logic with Engineering Applications: McGraw Hill, 1995.

[11] B. Kosko, Neural Networks and Fuzzy Systems: A Dynamic Systems Approach to Machine Intelligence. Englewood Cliffs, NJ: Prentice Hall, 1992.
[12] R. Yager, "A New Methodology for Ordinal Multiobjective Decisions Based on Fuzzy Sets," Decision Science, vol. 12, pp. 589-600, 1981.

[13] L. A. Zadeh, "Fuzzy Sets," Information and Control, vol. 8, pp. 338-353, 1965

[14] H. L. Willis and H. Tram, "Tutorial on Spatial Load Forecasting,"IEEE, 1991.

[15] H. L. Willis, R. W. Powell, and H. N. Tram, "Computerized Methods for Analysis of Impact of Demand Side Management on Distribution Systems," IEEE Transactions on Power Delivery, pp. 1236, 1987.

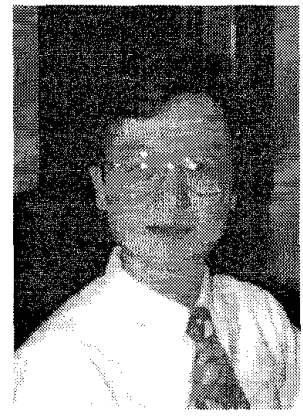

Mo-yuen Chow earned his B.S. degree at the University of Wisconsin-Madison (1982); M. Eng. degree (1983) and Ph.D. degrees at Cornell University (1987), all in Electrical Engineering. Upon completion of his Ph.D., Dr. Chow joined the faculty of North Carolina State University in Raleigh, NC, where he is presently an Associate Professor in the. Department of Electrical and Computer Engineering.

Since 1987, Dr. Chow has been working as a Principal Investigator in several projects in the areas of system monitoring, fault detection and control, applications of artificial neural network and fuzzy logic to power engineering. Dr. Chow served as a guest editor of IEEE Transactions on Industrial Electronics Special Issue on Application of Intelligent Systems to Industrial Electronics. $\mathrm{He}$ is currently an Associate Editor of the IEEE Transactions on Industrial Electronics. He is also listed in Who's Who in Asian Americans.

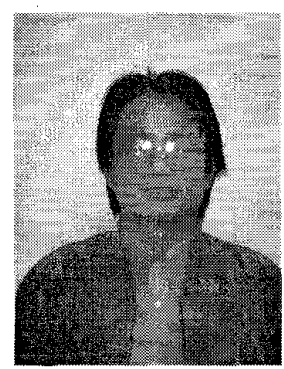

Jinxiang Zhu received his BSEE degree from Tsinghua University in Beijing PRC, and his MSEE degree from North Carolina State University in Electrical Engineering, respectively in 1990 and 1994. Currently he is Ph.D. candidate in North Carolina State University. He is student member of IEEE. His research interests include power system operation and planning, optimization techniques, fuzzy logic, and neural networks.

Hahn Tram, IEEE Senior Member, is Senior Principal Consultant of Distribution Information Systems, ABB Power T\&D Company Inc.. His sixteen years experience includes development work in power distribution load forecasting, substation and feeder optimization, and volt/var correction. He was an original developer of ABB's CADPAD ${ }^{(8)}$ software. Mr. Tram has consulted with utilities worldwide for distribution planning and engineering. He has BSEE and MSEE from Texas A\&M University, and further studies at Purdue University and Carnegie Mellon University. 